\title{
Viewpoint \\ Structure of HER receptors and intracellular localisation of downstream effector elements gives insight into mechanism of tumour growth promotion
}

Caroline J Witton

Endocrine Cancer Group, University of Glasgow, UK

Corresponding author: Caroline J Witton (e-mail: cjw6r@clinmed.gla.ac.uk)

Published: 30 May 2003

Breast Cancer Res 2003, 5:206-207 (DOI 10.1186/bcr613)

(C) 2003 BioMed Central Ltd (Print ISSN 1465-5411; Online ISSN 1465-542X)

\section{Introduction}

Since the discovery that the receptor HER2 (erbB2/neu) was overexpressed in $20-30 \%$ of breast cancers, and its subsequent association with poor prognosis, there has been much interest in this protein. The production of the humanised antibody to HER2 (Herceptin [trastuzumab]) and the use of Herceptin to treat patients with HER2 positive tumours has emphasised the potential of targeted treatments for breast cancer. The differences between HER2 and the other members of the type I receptor family (epidermal growth factor receptor [EGFR], HER3, HER4) have also been researched. HER2 differs in that it has no known ligand and when overexpressed HER2 can form dimers. In contrast the other receptors have several known ligands that promote dimer formation on binding. HER receptors can form both homo and heterodimers with HER2 acting as the preferred dimer partner. The activation of these receptors either by overexpression or ligand binding leads to the activation of intracellular growth promoting pathways and thus promotes tumour growth.

\section{Structure of HER receptors and localisation of downstream elements}

A recent paper in Nature [1] reports the crystal structure of the extracellular region of HER2 alone and in combination with Herceptin. HER receptors comprise an extracellular region of about 630 amino acids, a single membrane-spanning region and a cytoplasmic tyrosine kinase. The extracellular region contains four domains arranged as a tandem repeat of a two-domain unit consisting of a $~ 190$-amino acid $L$ domain (domains I and III) followed by a 120-amino acid cysteine-rich domain (domains II and IV) [2]. Crystal structures of HER receptors complexed to epidermal growth factor (EGF) or transforming growth factor (TGF)- $\alpha$, reveal these ligands bound to domains I and III, and a long finger-like projection from domain II mediating an inter-receptor dimer. Crystal forms of non-activated forms of HER3 [2] and HER1 (EGFR) [3] reveal this finger-like projection from domain II making an intramolecular contact with domain IV preventing dimerisation by occluding the dimerisation interface. Binding of ligand must therefore cause a domain rearrangement, bringing domains I and III close together and freeing the domain II projection to participate in dimer formation. HER2 lacks the domain II-IV contact and the domain I-III interface appears stable [1]. The HER2 domain arrangement is thus very similar to that observed in other HER receptors when complexed with ligand. The fact that HER2 lacks the internal autoinhibitory structure may explain why it readily forms dimers in the absence of ligand; in effect it is in the active configuration at all times.

Three papers in Nature Medicine [4,5,6] give an insight into one way that active HER receptors may promote tumour growth. Active receptors can stimulate the phosphatidyl inositol $3^{\prime}$-kinase ( $\left.\mathrm{P} \mid 3 \mathrm{~K}\right)$ signalling pathway via binding of the p85 subunit of PI3K resulting in activation of Akt. The Akt kinase inactivates several pro-apoptosis molecules including Bad, caspase-9, forkhead transcription factors, $1 \kappa \beta$ kinase and p53 (via MDM2 mediated phosphorylation). All three papers describe the translocation of p27 to the cytoplasm when phosphorylated by Akt. This removes the inhibition of the cyclin dependant kinase, cyclin-E/CDK2, mediated by nuclear p27. CDK2 is involved in G1 arrest so this inhibition increases cell proliferation. Cytoplasmic p27 was demonstrated in $41 \%$ of breast cancers $[4,5,6]$ along with activated Akt and this correlated with poor prognosis [6]. In normal breast tissue activated Akt was not detected and p27 was nuclear. The potential link between this mechanism and HER2 activa- 
tion was demonstrated by Shin et al [5] who decreased activation of Akt by blocking HER2 with Herceptin, resulting in increased levels of p27 in the nucleus. Akt can also be activated via other receptors, and also by Ras activation, mutational loss of PTEN or activating mutation of Akt itself, all of which could lead to translocation of p27 and growth stimulation. Liang et al [6] demonstrated that activation of Akt renders cells resistant to growth inhibition by TGF- $\beta$ and interleukin- 6 , and that inhibition of the Akt pathway reverses this effect.

\section{Conclusion}

The first 3 papers are of note as they demonstrate the importance of structural studies to elucidate the action of proteins. They demonstrate a structural difference between HER2 and other human epidermal growth factor receptors (EGFR and HER3) that suggests reasons why HER2 actively promotes tumour growth. The latter three papers demonstrate the importance of Akt in breast cancer with its ability to remove $\mathrm{G} 1$ arrest via phosphorylation of p27. This can be as a result of stimulation by receptors (e.g. HER overexpressors) or by other Akt stimulatory mechanisms. These papers raise several possibilities for breast cancer therapies; the blocking of Akt could make cancer cells more sensitive to many growth inhibitory agents. Designing drugs to target the regions of HER receptors required for binding may effectively block some Akt stimulation and therefore tumour growth. Also, cytoplasmic localisation of p27 could serve as a marker of HER receptor activation, which may aid in treatment of HER2 positive tumours. Currently the presence of HER2 overexpression and/or gene amplification is tested for rather than activity of the receptor and that may in some part explain the failure of Herceptin in some patients. These papers provide more information about specific growth promotion mechanisms in breast tumours and could help towards producing genetically targeted therapies appropriate to what drives growth in individual breast tumours.

\section{Competing interests}

None declared.

\section{References}

1. Cho HS, Mason K, Ramyar KX, Stanley AM, Gabelli SB, Denney DW, Leahy DJ:. Structure of the extracellular region of HER2 alone and in complex with the Herceptin Fab. Nature 2003, 421:756-760.

2. Cho HS, Leahy DJ: Structure of the extracellular region of HER3 reveals an interdomain tether. Science 2002,297:13301333.

3. Ferguson KM, Berger MB, Mendrola JM, Cho HS, Leahy DJ, Lemmon MA: EGF activates its receptor by removing interactions that autoinhibit ectodomain dimerization. Molecular Cell 2003, 11:507-517.

4. Viglietto G, Motti ML, Bruni P, Melillo RM, D'Alessio A, Califano D, Vinci F, Chiapetta G, Tsichlis P, Bellacosa A, Fusco A, Santoro M: Cytoplasmic relocalization and inhibition of the cyclin-dependant kinase inhibitor p27(Kip1) by PKB/Akt-mediated phosphorylation in breast cancer. Nat Med 2002, 8:1136-1144.
5. Shin I, Yakes FM, Rojo F, Shin NY, Bakin AV, Beselga J, Arteaga CL: PKB/Akt mediates cell-cycle progression by phosphorylation of p27(Kip1) at threonine 157 and modulation of its celIular localization. Nat Med 2002, 8:1145-1152.

6. Liang J, Zubovitz J, Petrocelli T, Kotchetkov R, Connor MK, Han K, Lee JH, Ciarallo S, Catzavelos C, Beniston R, Franssen E, Slingerland JM: PKB/Akt phosphorylates p27, impairs nuclear import of p27 and opposes p27-mediated G1 arrest. Nat Med 2002, 8:1153-1160.

\section{Note}

This article is based on papers highlighted by Faculty of 1000 (http://www.facultyof1000.com/start.asp), a web-based literature awareness service. Faculty of 1000 evaluations available for articles cited in this report may be viewed at: http://www.breast-cancer-research.com/reports/bcr613.asp

\section{Correspondence}

Caroline J Witton, Endocrine Cancer Group, University of Glasgow, Division of Cancer Sciences and Molecular Pathology Surgery, L2 QE Building, Royal Infirmary, Glasgow G312ER, UK. Tel/fax: +44 (0)141 211 5432; e-mail: cjw6r@clinmed.gla.ac.uk 